\title{
Anatomic Landmarks and Morphometric Measurements for Accurate Localization of the Sphenopalatine Ganglion via the Transnasal and Infrazygomatic Approaches: A Cadaveric Study
}

\author{
${ }^{1}$ Tulika Gupta, ${ }^{2}$ Anjali Aggarwal, ${ }^{3}$ Daisy Sahni
}

\begin{abstract}
Objective: Sphenopalatine ganglion (SPG) block is used for a variety of craniofacial pain syndromes either through the transnasal route or via the infrazygomatic approach. Intraoperative imaging can identify the pterygopalatine fossa (PPF) but not the exact position of the SPG. Accurate localization of the SPG requires knowledge of the relevant anatomical landmarks.
\end{abstract}

Materials and methods: Thirty mid sagittal head and neck cadaveric sections were studied and the morphometric data was collected to facilitate correct SPG localization via trans-nasal approach and infrazygomatic approach.

Results: The sphenopalatine foramen (SPF) was located at an average distance of $55 \mathrm{~mm}$ from the anterior nasal spine at a mean angle of $22^{\circ}$. It was at or just superior to the midpoint of a line joining the skull base and the hard palate. The SPG was located $4 \mathrm{~mm}$ posterior and $4.7 \mathrm{~mm}$ lateral to the SPF at a mean distance of $6.3 \mathrm{~mm}$ from the SPF at an inclination of about $50^{\circ}$ in both the sagittal and coronal planes. In the infrazygomatic approach, the SPG was between 4.5 and $6.3 \mathrm{~cm}$ from the skin and an angle of about $7^{\circ}$ posterior and inferior.

Conclusion: These morphometric measurements will be of help to the clinician for accurate electrode or needle placement for SPG block and in avoiding complications related to inaccurate needle placement.

Keywords: Sphenopalatine block, Sphenopalatine foramen, Sphenopalatine ganglion, Endonasal approach Infrazygomatic approach.

How to cite this article: Gupta T, Aggarwal A, Sahni D. Anatomic Landmarks and Morphometric Measurements for Accurate Localization of the Sphenopalatine Ganglion via the Transnasal and Infrazygomatic Approaches: A Cadaveric Study. J Postgrad Med Edu Res 2014;48(1):14-18.

Source of support: Nil

Conflict of interest: None

\section{INTRODUCTION}

The sphenopalatine ganglion (SPG) is a parasympathetic ganglion in the course of greater petrosal nerve and is

\footnotetext{
${ }^{1,2}$ Assistant Professor, ${ }^{3}$ Professor

${ }^{1-3}$ Department of Anatomy, Postgraduate Institute of Medical Education and Research, Chandigarh, India
}

Corresponding Author: Tulika Gupta, Assistant Professor Department of Anatomy, Postgraduate Institute of Medical Education and Research, Chandigarh, India, Phone: 01722755203, e-mail: tulikag11@yahoo.com located in the pterygopalatine fossa (PPF). This ganglion has multiple connections to trigeminal, facial and sympathetic systems, consists of somatosensory, sympathetic as well as parasympathetic fibers and receives a sensory, parasympathetic and a sympathetic root. ${ }^{1-4}$ Sphenopalatine ganglion block is an accepted and effective method for treatment of variety of clinical conditions, such as cluster headaches, acute migraine, trigeminal and other facial neuralgias, pain relief in head and neck malignancies as well as amelioration of musculoskeletal pain. ${ }^{1,2,4-9}$ The SPG blockade has been achieved with variety of methods. Commonly, the SPG is targeted by needle or electrode placement via the transnasal route through the sphenopalatine foramen (SPF) or percutaneously by an infrazygomatic approach. ${ }^{10-14}$ Pterygopalatine fossa (PPF) anatomy has been described by many authors ${ }^{13,15-17}$ but we could not come across any study giving detailed morphometric parameters to locate the SPG via different approaches. For the therapy to be effective and safe, accurate localization of the SPG is mandatory. Thus, the present study was designed to identify anatomical landmarks which would help the clinicians in accurate targeting of the SPG. This would also help in avoiding complications related to incorrect placement of needles.

\section{MATERIALS AND METHODS}

The study was conducted on 30 mid sagittal sections of the head and neck. These cadaveric specimens were from adult North West Indian population, available in the Department of Anatomy, PGIMER. Transnasal and per cutaneous infrazygomatic approaches were used to localize the ganglion. The specimens were fixed in anatomical position to take various measurements. The metrical parameters were taken with the help of a digital vernier caliper accurate up to $0.02 \mathrm{~mm}$. This study has been approved by the institute ethical committee.

\section{Transnasal Approach to the SPG}

Localization of the SPF (Fig. 1): the SPF is located near the posterior attachment of the middle turbinate; just above the 
ethmoidal crest. The posterior end of the attached margin of the middle turbinate was reflected down to find the SPF beneath the mucosa. Sphenopalatine artery was seen emerging out of the SPF.

The following parameters were taken:

- The distance between the SPF and the anterior nasal spine.

- The shortest vertical distance from the skull base to the center of the SPF.

- The shortest vertical distance from the hard palate to the center of the SPF.

These three distances provide coordinates for correct localization of the SPF.

- The angle between the hard palate and the line joining the anterior nasal spine with the SPF was measured at the anterior nasal spine.

Dissection to expose the SPG: the perpendicular plate of the palatine bone was identified and drilled to open the pterygopalatine fossa (PPF), while preserving the SPF (Fig. 2). The SPG was identified after the dissection. Sphenopalatine ganglion was present in the posterior aspect of the fossa while the maxillary artery was seen anteriorly. Following parameters were measured (Fig. 3A is an orientation figure):

- The direct distance between the SPF and the center of the SPG (Fig. 3B).

- The anteroposterior displacement between the SPF and the center of the SPG (Fig. 3C).

- The lateral displacement from the SPF to the center of the SPG.

- The angle of the needle/endoscope (passing through the sphenopalatine foramen to reach the center of the SPG) trajectory to the sagittal plane passing through the SPF (Fig. 3D).

\section{Percutaneous Infrazygomatic Approach to the SPG}

Point of needle insertion: A point was marked at the anterior end of the zygomatic arch on the inferior border. The skull was fixed in anatomical position. A long needle was inserted percutaneously till it reached the PPF through the pterygomaxillary fissure (PMF). The accuracy of needle placement was confirmed by dissection. The following data was recorded:

- The angle of needle trajectory was measured in respect to the transverse plane and the coronal plane, passing through the point of needle insertion.

- The distance from the point of needle insertion up to the PMF.

- The distance from the point of needle insertion up to the SPG.

\section{RESULTS}

\section{Intranasal Approach to the Pterygopalatine Ganglion}

1. Position of the SPF in the nasal cavity: the SPF was located at an average distance of $55 \mathrm{~mm}$ from the anterior nasal spine. In most of the specimens, this value was between 50 and $60 \mathrm{~mm}$. The mean value of the angle of the line joining the anterior nasal spine and the foramen, with the hard palate was found to be $22 \mathrm{~mm}$. In about $70 \%$ of cadavers, this angle was $20^{\circ}$. The relative position of the SPF in relation to its distance from the hard palate

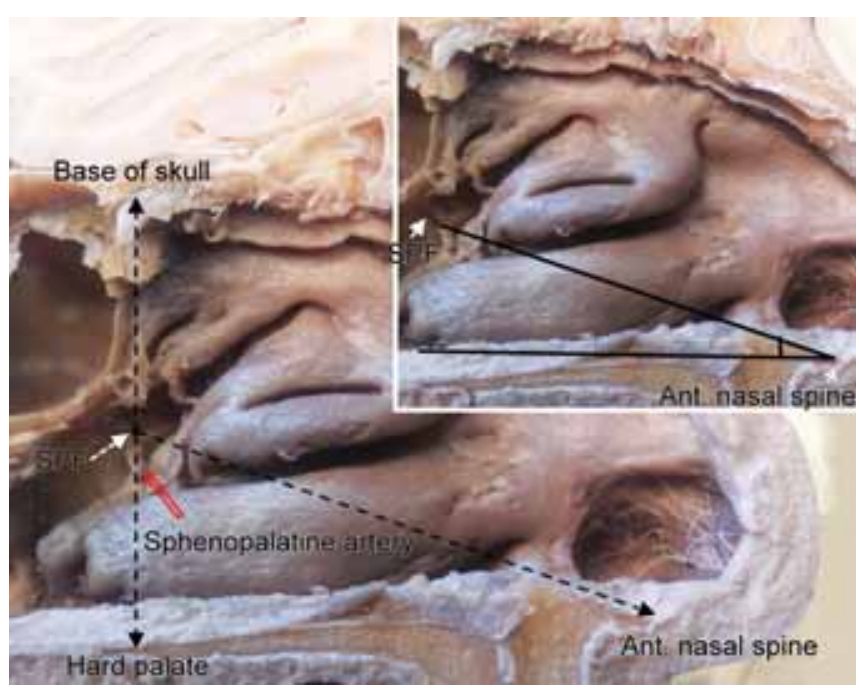

Fig. 1: Depiction of the shortest distances measured from the sphenopalatine foramen (SPF) to the anterior nasal spine (ANS), the hard palate and the base of the skull. The sphenopalatine artery can be seen emerging from the SPF. Inset shows the angle measured at the ANS, between the hard palate and line joining the ANS and SPF

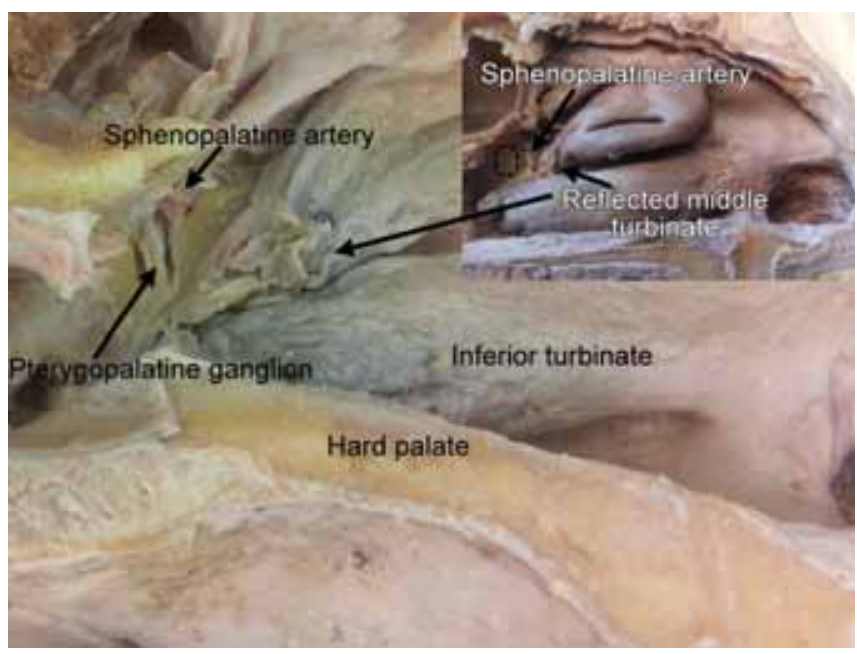

Fig. 2: The attached posterior end of the middle turbinate has been reflected down to expose the perpendicular plate of palatine bone. In the inset dotted area on the perpendicular plate of palatine bone marks the bone which was drilled. The sphenopalatine ganglion was exposed, while preserving the sphenopalatine foramen and the sphenopalatine artery emerging from the foramen 

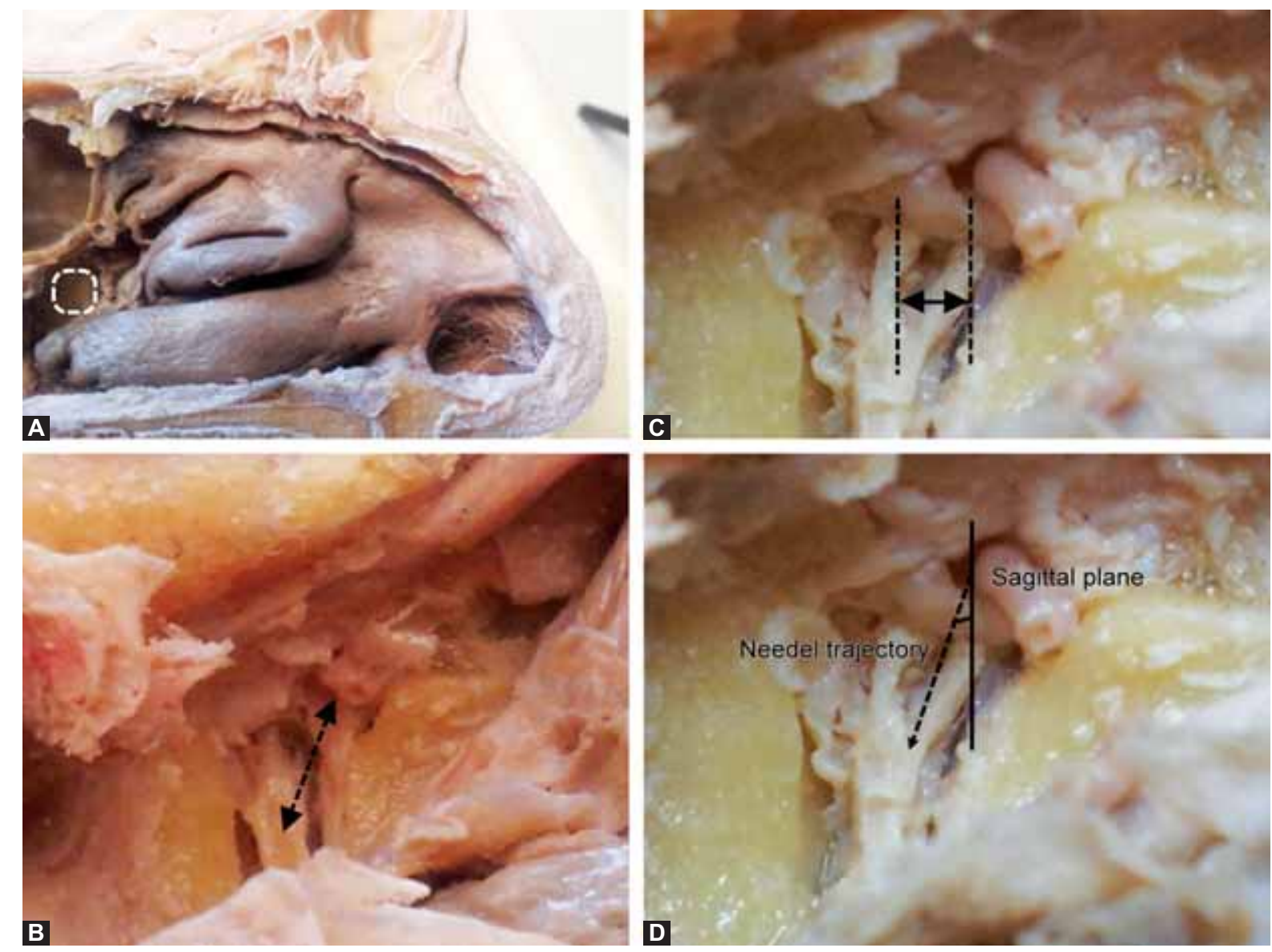

Figs 3A to D: (A) It is an orientation figure. Area marked on perpendicular plate show the perpendicular plate of palatine bone which was drilled to open the pterygopalatine fossa and expose the sphenopalatine ganglion (SPG); (B) Solid line with arrows depicts the antero-posterior displacement between the sphenopalatine foramen (SPF) and SPG; (C) Anatomical distance between the SPF and SPG is marked; (D) The figure shows angle of the needle/endoscope (passing through the sphenopalatine foramen to reach the center of the sphenopalatine ganglion) trajectory to the sagittal plane passing through the SPF

as well as from the base of the skull was analyzed. The measurements show that the foramen was located at or just superior to the midpoint of the distance between the hard palate and the base of the skull in most of the cases (Table 1).

2. Position of the SPG in relation to the SPF (Table 2): The mean of the shortest distance from the center of the foramen to the nearest point of the SPG was found to be $6.3 \mathrm{~mm}$. In three-dimensional positioning, the SPG was on an average $4 \mathrm{~mm}$ posterior and $4.7 \mathrm{~mm}$ lateral to the SPF. If one passes an endoscope through the foramen to the ganglion, the endoscope would subserve an angle of $48.3^{\circ}$ with the coronal plane and $51.7^{\circ}$ with the sagittal plane. In practical terms, a line joining the center of the foramen to the ganglion was inclined about $50^{\circ}$ in both the sagittal and the coronal planes.

\section{Localization of the SPG via the Infrazygomatic Approach (Table 3)}

The distance of the PMF from the skin surface from the entry point of the needle ranged roughly between 4 and
$5.5 \mathrm{~cm}$ (mean $4.5 \mathrm{~cm}$ ) while the SPG was located at a depth of 4.5 to $6.3 \mathrm{~cm}$ (mean $5.3 \mathrm{~cm}$ ). The angle of the needle when it touched the ganglion was $7^{\circ}$ with the coronal plane (inferiorly) and $7^{\circ}$ with the transverse plane (posteriorly). These two angles were remarkable in being almost same for all specimens in both the measured planes.

\section{DISCUSSION}

Sphenopalatine ganglion is commonly targeted either through the transnasal approach or via the percutaneous infrazygomatic technique. The transnasal injection technique, either blindly or with the assistance of a speculum, carries the danger of nasal mucosa sloughing during needle insertion. ${ }^{12}$ This led to the development of the transnasal endoscopic technique in which the topical block, or needle infiltration, was performed under direct vision using rigid sinuscope. ${ }^{10,13}$

It is well documented that drug delivery directly in the vicinity of the SPG is more effective and accurate in pain relief than instillation of the drug in the middle meatus 
mucosa. ${ }^{12,14,18}$ In the transnasal endoscopic approach, the SPF is visualized behind the middle turbinate and a needle is inserted through this foramen into the PPF for injection of anesthetic drugs for SPG block. ${ }^{4}$

The infrazygomatic approach is necessary to perform the majority of neurolysis techniques. The infrazygomatic approach has been commonly performed blindly in the past. In this technique, the needle is inserted under the zygomatic arch and advanced through the PMF to reach the PPF which houses the SPG. In recent years, the approach is carried out by using image guidance and a direct lateral approach to place a canula into the superior portion of the PPF without requiring local anesthetic to diffuse across mucous and bony membranes and does not rely on secondary spread via the nerves of the greater palatine canal. ${ }^{3,11}$

Most clinicians prefer to use intraoperative fluoroscopic or CT guidance for accurate localization of the PPF for either drug delivery or for RF lesioning. Intraoperative imaging can depict the bony landmarks of PPF but not the precise location of the SPG in the fossa. Besides, knowledge of the approximate position of the SPG via different surgical routes will be extremely helpful in its localization with imaging guidance.

Our results show that the SPF was consistently located either midway or 2 to $3 \mathrm{~mm}$ superior to the midpoint of a line joining the hard palate and the floor of the anterior fossa just behind the posterior attached end of the middle turbinate. It was also observed that a needle passing though the SPF would have to be inclined at an angle of about $50^{\circ}$ in both coronal and sagittal planes to reach the SPG. Passing through the SPF at this angle, the SPG was found to be located at a mean distance of $6.3 \mathrm{~mm}$ and about 4 to $5 \mathrm{~mm}$ posterior and 4 to $5 \mathrm{~mm}$ lateral to the SPF.

Anatomical measurements for the infrazygomatic technique demonstrated that a needle passed through the skin below the zygomatic bone at the junction of its body and arch should be inclined $7^{\circ}$ posteriorly and $7^{\circ}$ inferiorly and advanced to a depth of 4.5 to $6.3 \mathrm{~cm}$ to reach the SPG. The direction of the needle was found to be remarkable consistent in most of the specimens.

The PPF has branches of the maxillary artery and in addition to the various nerves in relation to the SPG. Injury to the vessels can cause postoperative hematoma formation while numbness and hypoesthesia of the soft palate have been described due to injury to the greater palatine nerve. ${ }^{16,19,20}$ Maxillary hyperpathia following RF lesion of the SPG has also been reported and is possibly the consequence of a partial peripheral injury of the maxillary nerve causing a deafferentation pain. The best way to avoid these complications is accurate needle placement. ${ }^{19}$

There is adequate literature available of the detailed anatomy of the SPG as well as the PPF. However, morphometric data regarding localization of the SPG within the PPF via the commonly used routes is not available. The data obtained from this cadaveric study define landmarks that provide safe entrance into PPF via transnasal and

Table 1: Morphometric measurements for localization of the SPF

\begin{tabular}{llccc}
\hline Parameters & Mean & Standard deviation & Minimum & Maximum \\
\hline Distance from ANS to SPF & 55.1 & 4.5 & 46.3 & 60.6 \\
Angle at ANS & $21.9^{\circ}$ & $3.2^{\circ}$ & $20^{\circ}$ & $30^{\circ}$ \\
Distance from the HP & 24.6 & 3.0 & 18.4 & 28.4 \\
Distance from the base of skull & 21.9 & 3.8 & 14.0 & 27.3 \\
\hline
\end{tabular}

ANS: Anterior nasal spine; SPF: Sphenopalatine foramen; HP: Hard palate; All metrical measurements are in millimeter

Table 2: The sphenopalatine ganglion (SPG) in relation to the sphenopalatine foramen (SPF)

\begin{tabular}{llccc}
\hline Parameters & Mean & Standard deviation & Minimum & Maximum \\
\hline Antero-posterior displacement between SPF and SPG & 4.0 & 0.7 & 2.7 & 5.9 \\
Lateral displacement between SPF and SPG & 4.7 & 1.2 & 2.8 & 7.3 \\
Anatomical distance between SPF and SPG & 6.3 & 1.9 & 3.4 & 10.6 \\
Angle with the coronal plane & $48.3^{\circ}$ & $4.1^{\circ}$ & $40^{\circ}$ & $50^{\circ}$ \\
Angle with the sagittal plane & $51.7^{\circ}$ & $4.1^{\circ}$ & $50^{\circ}$ & $60^{\circ}$ \\
\hline
\end{tabular}

All metrical measurements are in millimeter

Table 3: Localization of the SPG via the infrazygomatic approach

\begin{tabular}{llccc}
\hline Parameters & Mean & Standard deviation & Minimum & Maximum \\
\hline Distance from skin to the PMF & 44.8 & 5.0 & 39.9 & 54.3 \\
Distance from skin to the SPG & 52.9 & 5.6 & 44.9 & 63.4 \\
Angle of the needle with transverse plane & $7.3^{\circ}$ & $1^{\circ}$ & $7^{\circ}$ & $10^{\circ}$ \\
Angle of the needle with coronal plane & $6.9^{\circ}$ & $0.6^{\circ}$ & $6^{\circ}$ & $8^{\circ}$ \\
\hline
\end{tabular}

All metrical measurements are in millimeter 
percutaneous infrazygomatic approaches. It is hoped that the measurements and parameters detailed in this study would help the clinician in precise lesion making or targeting and thus minimize the incidence of complications.

\section{REFERENCES}

1. Peterson JN, Schames J, Schames M, et al. Sphenopalatine ganglion block: a safe and easy method for the management of orofacial pain. Cranio 1995;13:177-181.

2. Day M. Neurolysis of the trigeminal and sphenopalatine ganglions. Pain Pract 2001;1:171-182.

3. Yin W. Sphenopalatine ganglion radiofrequency lesions in the treatment of facial pain. Tech Reg Anesth Pain Manag 2004;8: 25-29.

4. Piagkou M, Demesticha T, Troupis T, et al. The pterygopalatine ganglion and its role in various pain syndromes: from anatomy to clinical practice. Pain Pract 2012;12(5):399-412.

5. Lebovits AH, Alfred H, Lefkowitz M. Sphenopalatine ganglion block: clinical use in the pain management clinic. Clin J Pain 1990;6:131-136.

6. Sluder G. The control of earache through the nasal ganglion. JAMA 1992;78:1708.

7. Ferrante M, Kaufman A, Dunbar S, et al. Sphenopalatine ganglion block for the treatment of myofascial pain of the head, neck and shoulders. Reg Anaesth Pain Med 1998;23:30-36.

8. Rosenberg M, Phero JC. Regional anesthesia and invasive techniques to manage head and neck pain. Otolaryngol Clin North Am 2003;36:1201-1219.

9. Windsor R, Jahnke S. Sphenopalatine ganglion blockade: a review and proposed modification of the transnasal technique. Pain Physician 2004;7:283-286.
10. Prasanna A, Murthy PS. Sphenopalatine ganglion block under vision using rigid nasal sinuscope. Reg Anesth 1993;18:139-140.

11. Stechison MT, Brogan M. Transfacial transpterygomaxillary access to foramen rotundum, sphenopalatine ganglion and the maxillary nerve in the management of atypical facial pain. Skull Base Surg 1994;4:15-20.

12. Cambareri JJ. Sphenopalatine ganglion. In: Thomas PS, editor. Image guided pain management. Philadelphia: Lippincott Raven Publishers 1997;27-33.

13. Alfieri A, Jho HD, Schettino R, et al. Endoscopic endonasal approach to the pterygopalatine fossa: anatomic study. Neurosurgery 2003;52(2):374-378.

14. Yang IY, Oraee S. A novel approach to transnasal sphenopalatine ganglion injection. Pain Physician 2006;9:131-134.

15. Li J, Xu X, Wang J, et al. Endoscopic study for the pterygopalatine fossa anatomy: via the middle nasal meatus-sphenopalatine approach. J Craniofac Surg 2009;20:944-947.

16. Rusu MC, Pop F, Podoleanu L, et al. The pterygopalatine ganglion in humans: a morphological study. Ann Anat 2009;191: 196-202.

17. Oomen KPQ, Pameijer FA, Zwanenburg JJM, et al. Improved depiction of pterygopalatine fossa anatomy using ultrahighresolution magnetic resonance imaging at 7 tesla. The Scientific World Journal 2012:691095.

18. Felisati G, Arnone F, Lozza P, et al. Sphenopalatine endoscopic ganglion block: a revision of a traditional technique for cluster headache. Laryngoscope 2006;116(8):1447-1450.

19. Sanders M, Zuurmond WW. Efficacy of sphenopalatine ganglion blockade in 66 patients suffering from cluster headache: a 12- to 70-month follow-up evaluation. J Neurosurg 1997;87(6):876-880.

20. Bolger WE, Osenbach R. Endoscopic transpterygoid approach to the lateral sphenoid recess. Ear Nose Throat 1999;78:36-46. 